\title{
Cell optoporation with a sub-15 fs and a 250-fs laser
}

Hans Georg Breunig

Ana Batista

Aisada Uchugonova

Karsten König 


\section{Cell optoporation with a sub-15 fs and a 250 -fs laser}

\author{
Hans Georg Breunig, ${ }^{a, *}$ Ana Batista, a,b Aisada \\ Uchugonova, ${ }^{a, b}$ and Karsten König ${ }^{a, b}$ \\ a JenLab GmbH, Science Park 2, Campus D 1.2, 66123 Saarbrücken, \\ Germany and Schillerstr. 1, 07745 Jena, Germany

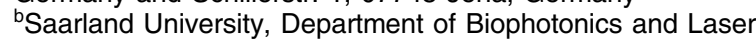 \\ Technology, Campus A5.1, 66123 Saarbrücken, Germany
}

\begin{abstract}
We employed two commercially available femtosecond lasers, a Ti:sapphire and a ytterbium-based oscillator, to directly compare from a user's practical point-of-view in one common experimental setup the efficiencies of transient laser-induced cell membrane permeabilization, i.e., of so-called optoporation. The experimental setup consisted of a modified multiphoton laser-scanning microscope employing high-NA focusing optics. An automatic cell irradiation procedure was realized with custom-made software that identified cell positions and controlled relevant hardware components. The Ti:sapphire and ytterbium-based oscillators generated broadband sub-15-fs pulses around $800 \mathrm{~nm}$ and 250-fs pulses at $1044 \mathrm{~nm}$, respectively. A higher optoporation rate and posttreatment viability were observed for the shorter fs pulses, confirming the importance of multiphoton effects for efficient optoporation. ๑ 2016 Society of Photo-Optical Instrumentation Engineers (SPIE) [DOI: 10.1117/1.JBO.21.6.060501]
\end{abstract}

Keywords: cell transfection; optoporation; femtosecond laser; laserassisted transfection; laser assisted poration; $\mathrm{CHO}$ cells.

Paper 160200LR received Mar. 30, 2016; accepted for publication May 5, 2016; published online Jun. 1, 2016.

\section{Introduction}

An important as well as critical part for the investigation and manipulation of cells is to bring macromolecules through the cell membrane, which is normally impenetrable for larger molecules, into the cell's interior. The process allows to directly influence the cell state and later development in a desired way, but it may also harm or even lead to cell death. Several methods to overcome the cell membrane have been developed. ${ }^{1-3}$ A not-yetwidely-used technique is laser-assisted cell membrane poration (optoporation), which is based on transient permeabilization of the membrane by focused laser radiation. ${ }^{4-12}$ This method provides, very high poration and transfection rates as well as high posttreatment cell viability. There is even evidence that complex cell transformations, which are highly unlikely to occur with common methods such as optical reprogramming, take place with higher probability and within shorter time. ${ }^{13,14}$ Furthermore, optoporation is highly selective, reproducible,

*Address all correspondence to: Hans Georg Breunig, E-mail: breunig@ jenlab .de free of carrier substances, and their possible side effects as well as comparably easy to realize with common optical setups. ${ }^{7}$ Usually, nonmoving individual cells are manually targeted one by one for laser-assisted cell-membrane poration, which limits the number of cells that are addressable in a reasonable time. Drastic increases in the number of targetable cells have been achieved by approaches to completely automate the laser irradiation process or by targeting of nonadherent cells in a flow-through chamber. ${ }^{9}$ However, a priori the optimal experimental conditions are often not clear because of many different experimental parameters influencing the outcome as well as the difficulty to directly compare reported cell optoporation procedures, which have been realized with different cell types, different laser sources, and different optical setups, which complicates the direct comparison of the results. ${ }^{8}$ Cell posttreatment viability ratios and optoporation efficiencies vary in the literature from $50 \%$ to $100 \%$ and from $25 \%$ to $100 \%$, respectively. ${ }^{4,5,7}$ Some studies have attempted to pinpoint the effects of laser parameters such as pulse duration and mean power for one kind of cell in a common setup. The direct comparison of the literature results is, however, often hindered by different experimental setups and lasers used in different labs. ${ }^{6,8}$ Here, we compare from a practical point of view with an application-oriented user in mind two common, commercial off-the-shelf femtosecond oscillators, a typical short-broadband Ti:sapphire, and a more cost-effective ytterbium-based laser, in terms of achievable optoporation efficiency and posttreatment cell viability by employing them in the same experimental setup and treating cells, even from one common sample, with both lasers. Furthermore, a custommade software with hardware control was used to automate the laser-irradiation process. ${ }^{10}$ Differences in the experimental setups or preparation procedures that may influence results are in this way circumvented and the outcome can be regarded as resulting purely from the different laser characteristics.

\section{Material and Methods}

Two commercial femtosecond lasers were used for the laserassisted cell optoporation experiments. The first laser was a broadband Ti:sapphire oscillator (Integral, FemtoLasers); the second was a compact ytterbium-based femtosecond laser (HighQ-2, Newport Spectra-Physics). An overview of the laser parameters and some experimental settings is given in Table 1. Laser spectra are shown in Figs. 1(a) and 1(b). Note that both lasers generate pulses with different wavelengths, durations, and repetition rates.

The experimental setup, which has been described previously, ${ }^{15}$ is schematically shown in Fig. 1(c). It consists of a multiphoton imaging system (FemtoGene, JenLab $\mathrm{GmbH}$ ), which is based on a modified commercial microscope employing a laser-scanning setup, i.e., galvano-scanning mirrors, a beam expander, dichroic mirror, and a microscope objective. Chirped mirrors are used to precompensate for the group-velocity dispersion of the optics in case of the integral laser such that sub-15-fs-long pulses are present at the sample position, which was confirmed by autocorrelation measurements. For the optoporation, the laser-focus position was always kept fixed and the cells were centered on that position by a motorized $x-y$ microscope stage (Märzhäuser, SCAN IM $130 \times 100)$ ). A 1.3 NA 40× microscope objective (Zeiss, neofluar) was used with both lasers for focusing of the laser pulses and for imaging of the sample

$1083-3668 / 2016 / \$ 25.00$ (C) 2016 SPIE 
Table 1 Laser and experimental parameters.

\begin{tabular}{lcc}
\hline & HighQ-2 & Integral \\
\hline Spectral maximum (nm) & 1044 & 778,827 \\
Spectral width (FWHM) (nm) & 11 & 116 \\
In situ pulse duration (fs) & 250 & 15 \\
Repetition rate (MHz) & 63 & 85 \\
Power at sample (mW) & 62 & 15 \\
Pulse energy at sample (pJ) & 984 & 117 \\
Peak power at sample (kW) & 3.94 & 7.84 \\
Polarization direction & Vertical & Horizontal \\
Type of laser & Ytterbium based & Ti:sapphire \\
Size of laser housing (mm $\left.{ }^{3}\right)$ & $222 \times 204 \times 101$ & $498 \times 257 \times 80$ \\
Cooling & Air & Water \\
\hline
\end{tabular}

with a microscope camera. The vertical position of the objective was controlled by a piezoelectric positioning system (MIPOS, Piezosystem Jena $\mathrm{GmbH}$ ). A half-wave plate in the path of the HighQ-2 beam was used to establish equal linear polarization states of both lasers by changing the polarization direction of the HighQ-2 pulses to horizontal. By flipping a mirror, the output of one of the two lasers could be coupled into the microscope. The laser power at the sample was controlled by rotating a Glan-laser polarizer inside the common beam path. The laser exposure time was selected by setting the open duration of a fast mechanical shutter. Images were recorded with a 1.3-megapixel microscope camera (SPOT idea, SPOT Imaging Solutions). Microscope stage, camera, piezoelectric positioning system, and the optical beam shutter were all controlled by custom LabView-based software JL-CellFinder [elements indicated by dashed boundary line in Fig. 1(c)]. The software automatically identifies positions for irradiation by evaluating the image contrast, centers the positions in the laser focus by controlling the stage, and engages the laser irradiation by opening and closing the shutter. ${ }^{10}$ The exact location within the cell that is laser irradiated, i.e., a location on the cell membrane, cytoplasm, or nucleus, as well as the number of irradiated positions per cell strongly affects the optoporation efficiency and cell survival rate. ${ }^{8}$ About 2 to 4 positions per cell at or close to the membrane were targeted by the control software. The identification was done by image processing of bright-field microscope images of the sample and evaluation of simple image contrast changes evaluated along parallel lines. The algorithm enabled fast identification of a suitable position and, in combination with the control of hardware components, to irradiate several cells per second. ${ }^{10}$

The preparation of Chinese hamster ovary (CHO-K1) cells and the evaluation of the laser irradiation were performed as described previously. ${ }^{10}$ Briefly, cells were cultured in $35-\mathrm{mm}$ glass-bottom dishes with 500- $\mu \mathrm{m}$ grids, which were then placed directly on the microscope stage during the laser-irradiation procedure. To evaluate the outcome of the optoporation and cell viability, the cell samples were incubated together with the fluorescence dye diamidin-2-phenylindol (DAPI) shortly before the laser irradiation. After the laser irradiation, the cells were stained with calcein $\mathrm{AM}$ and ethidium bromide $(\mathrm{EtBr})$. The resulting cell fluorescence was evaluated with a fluorescence microscope (Zeiss Axio Observer D1 with LED module Colibri) about $30 \mathrm{~min}$ after the irradiation procedure. In case the DAPI

(a)

(b)
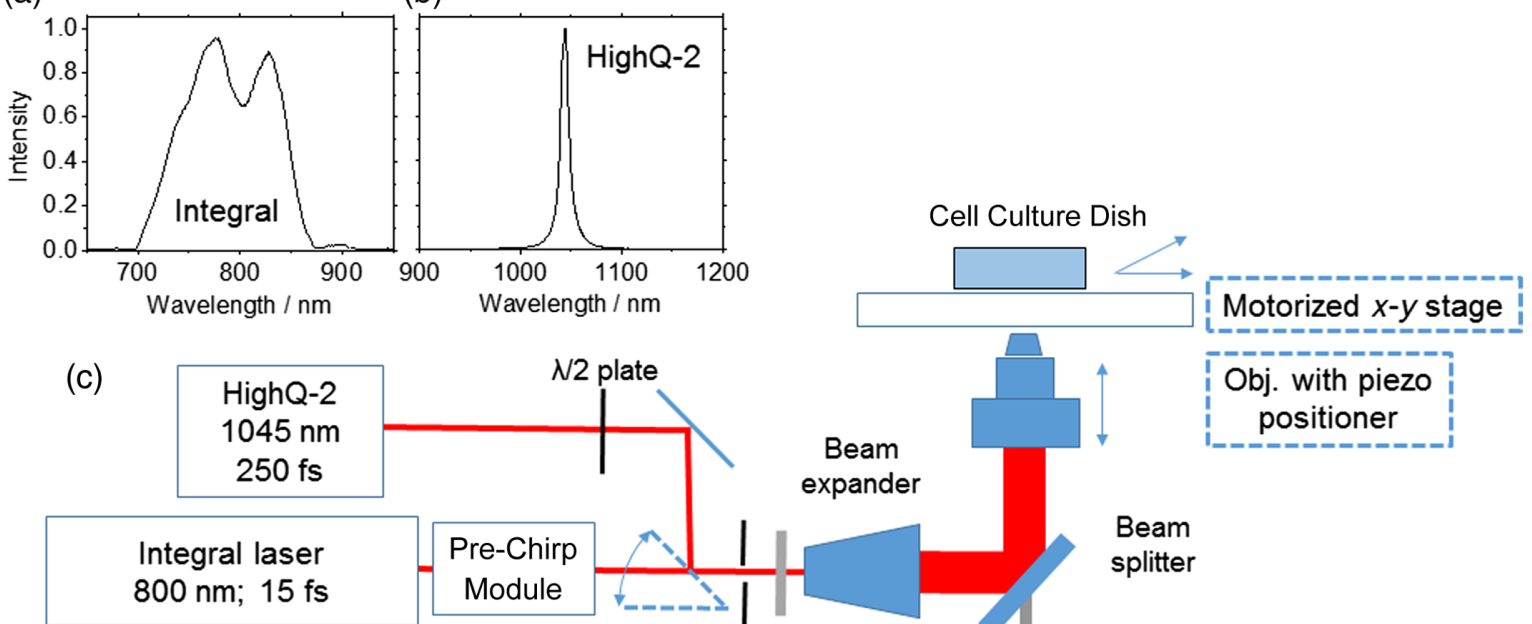

expand

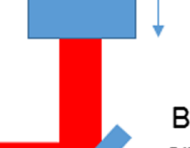

Beam

splitter

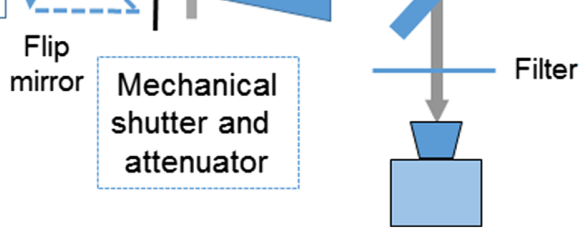

Camera

Fig. 1 Spectra of both lasers employed for the optoporation: (a) integral laser and (b) HighQ-2 laser. (c) Scheme of the experimental setup. The beam of one of the two lasers (HighQ-2 and integral) is guided to the sample depending on the position of the flip mirror. Stage scanning is used to position the sample for laser irradiation. Laser power and irradiation duration are controlled by the attenuator and shutter, respectively. The dashed rectangles indicate components controlled by the custom-made software. 
fluorescence intensity from a cell exceeded the intensity of the nonirradiated control samples by two times the standard deviation of the procedure, the cell was counted as positively optoporated. Based on calcein and EtBr fluorescence, the cell viability and posttreatment survival were determined. The ratios of optoporated and living as well as dead cells were normalized to the total number of irradiated cells. Cells that survived the laser irradiation and showed DAPI fluorescence above (below) the aforementioned threshold were regarded as optoporated (not optoporated) and living.

\section{Results and Discussion}

The ratio of optoporated and living cells as well as the portion of dead cells after the laser irradiation is shown in Fig. 2 as dependent on irradiation time (a and c) and total deposited energy (b and d), respectively. Each data point in Fig. 2 represents 20 to 50 irradiated cells from the same cell sample, i.e., from cells in different regions inside the same culture dish. The figure summarizes the main results of this letter. The mean power, pulse duration, and repetition rate were kept fixed at the values listed in Table 1. With increasing irradiation duration [Fig. 2(a)], the ratio of positively optoporated and living cells increases initially, then saturates, and decreases eventually. The saturation ranges differ for both lasers as well as the values of the maximum ratio. For irradiation durations exceeding about $60 \mathrm{~ms}$ for the HighQ-2 and about $120 \mathrm{~ms}$ for the integral laser, the fraction of optoporated and living cells does not increase further. A maximum of about $80 \%$ to $90 \%$ and of about $30 \%$ to $40 \%$ of optoporated and viable cells was achieved with the integral and HighQ-2, respectively. For longer irradiation durations, the ratio decreases for both lasers. The ratio of cells that did not survive the laser treatment is shown in Fig. 2(c). It increases steadily with longer irradiation duration even with increasing slopes. The increase is even steeper for the HighQ-2 than for the integral laser, reaching about $40 \%$ at $100 \mathrm{~ms}$ (HighQ-2) and $180 \mathrm{~ms}$ (integral). Cells in control samples without laser irradiation show a viability of close to $100 \%$ and zero positive optoporation. ${ }^{10}$

The data in Figs. 2(a) and 2(c) are shown in dependence on the irradiation duration, which is the experimentally directly accessible parameter. What is in fact varied is the total number of pulses applied to the cells during exposure and thereby the total energy deposited in the cell, which, however, is different for both lasers due to the different pulse repetition rates and applied mean powers, respectively. Figures 2(b) and 2(d) show the same experimental data as Figs. 2(a) and 2(c) in dependence on the total energy that is deposited during the laser exposure for both lasers. The ratio of optoporated and viable cells [Fig. 2(b)] exhibits a sharp maximum for the integral. A maximum is also present in the HighQ-2 data but is more spread out. The ratio of dead cells [Fig. 2(d)] increases for both lasers and is even steeper for the integral than for the HighQ-2 laser.

The initial increase in the ratio of optoporated and viable cells [Figs. 2(a) and 2(b)] is a result of the increasingly efficient optoporation, i.e., the membrane is more effectively permeabilized the more laser pulses reach it. At the same time, the laser radiation induces damage to the cells that eventually leads to direct or apoptosis-mediated cell death and thereby reduces the overall cell viability. This reduction of the overall cell viability with increasing irradiation duration leads eventually to the decrease of the ratio of optoporated and living cells that is shown in Fig. 2(a) and the increase of the curves shown in Fig. 2(c). The differences observed for the two lasers therefore can be attributed to the different laser characteristics, which differ in center wavelengths (spectrum), repetition rates, pulse durations, and pulse energies. This makes it difficult to attribute the differences to a single parameter. Rudhall et al. ${ }^{6}$ have
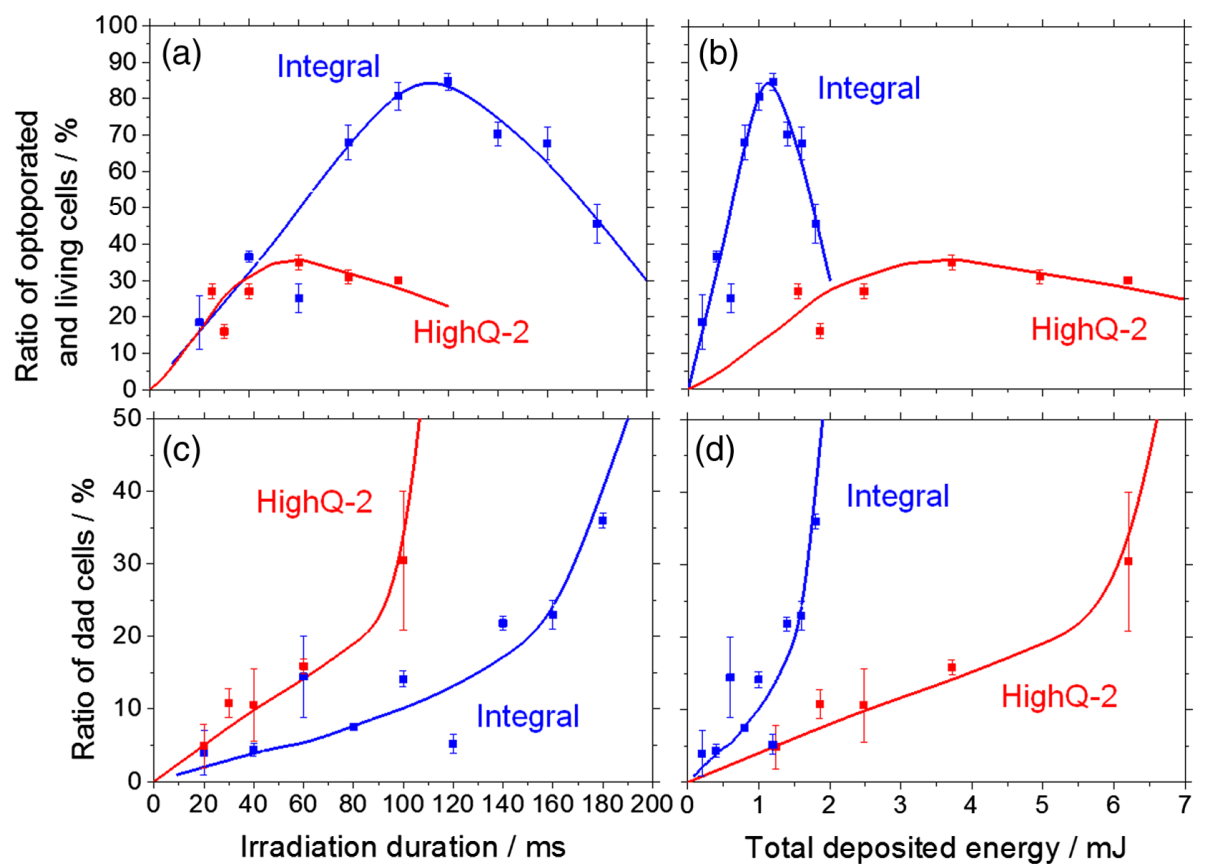

Fig. 2 Results of optoporation experiments with both lasers. (a, b) Ratio of optoporated and living cells in dependence on irradiation duration and on the total energy deposited into the irradiation cell region, respectively. (c, d) Ratio of dead cells/\% in dependence on irradiation time/ms and in dependence on the total energy deposited into the irradiation cell region, respectively. Each data point represents 20 to 50 irradiated cells. The lines are guides to the eye only. 
identified pulse duration, pulse energy, and number of pulses to all influence the optoporation. The importance of the different pulse durations for equal values of deposited energy during the laser irradiation is apparent in Figs. 2(b) and 2(d) as the most important parameter, which causes the differences observed with the integral and HighQ-2. The underlying process of the optoporation is multiphoton absorption that results in the creation of a plasma of quasi-free electrons, which in turn causes thermoelastic stress, chemical breakdown, and eventually a permeabilization of the cell membrane. ${ }^{6,16}$ Although the pulse duration and the associated peak power are more important for the underlying multiphoton processes than the total deposited energy, both parameters, peak power and total deposited energy, influence the cell viability, as can be seen by the different dependencies for both lasers in Fig. 2(d). However, the smaller pulse duration and the higher peak power of the integral pulses (Table 1) enable a more efficient membrane permeabilization at lower doses of the energy deposited in total, which results in a higher ratio of optoporated and viable cells compared to the HighQ-2 laser. Nevertheless, a sufficient number of pulses is required to enable efficient optoporation.

The influence of the different center wavelengths and spectral widths of the HighQ-2 and integral laser pulses is less significant than the influence of the different pulse durations in the case of purely nonresonant multiphoton generation of a plasma of quasifree electrons. ${ }^{16}$ However, the different center wavelengths could in principle lead to differing strong destructive nonlinear photochemical effects. Although thermal damage from multiphoton water absorption can be effectively ruled out for both lasers, genotoxic effects induced by three photon absorption of nucleic acid, which strongly absorbs below $290 \mathrm{~nm}$, can be expected to be more significant for the integral than for the longer wavelength of the HighQ-2 laser. ${ }^{17}$ It cannot be ruled out that this could contribute to the stronger increase of the ratio of dead cells observed in Fig. 2(d) for the integral compared to the HighQ-2 laser, although the irradiation was directed at the membrane only.

\section{Conclusion}

We compared two femtosecond lasers, which represent common commercially available laser classes, i.e., Ti:sapphire and ytterbium-based oscillators, in optoporation experiments with adherent $\mathrm{CHO}$ cells. Both lasers were coupled to the same experimental setup, which consisted of a laser scanning microscope employing a motorized microscope stage for sample positioning and control software for an automated cell-position selection and laser-irradiation procedure. The commercial Ti: sapphire and ytterbium-based lasers generated sub-15 fs and $250 \mathrm{fs}$ at center wavelengths of 800 and $1040 \mathrm{~nm}$, respectively. The shorter pulses resulted in significantly higher achievable optoporation efficiency and posttreatment cell viability.

\section{Acknowledgments}

We thank Dr. Tommi Hakulinen (High Q Laser GmbH), Professor Heinz Huber (Munich University of Applied Science), and Stefan Melzer (Newport Spectra-Physics GmbH) for the loan of the HighQ-2. Financial support from the Deutsche Forschungsgemeinschaft (DFG, Priority Programme 1327/2) is acknowledged.

\section{References}

1. K. A. Whitehead, R. Langer, and D. G. Anderson, "Knocking down barriers: advances in siRNA delivery," Nat. Rev. Drug Discovery 8(2), 129-138 (2009).

2. M. Yan et al., "A novel intracellular protein delivery platform based on single-protein nanocapsules," Nat. Nanotechnol. 5(1), 48-53 (2010).

3. R. Waehler, S. J. Russell, and D. T. Curiel, "Engineering targeted viral vectors for gene therapy," Nat. Rev. Genet. 8(8), 573-587 (2007).

4. U. K. Tirlapur and K. König, "Targeted transfection by femtosecond laser," Nature 418(6895), 290-291 (2002).

5. A. Uchugonova et al., "Targeted transfection of stem cells with sub-20 femtosecond laser pulses," Opt. Express 16(13), 9357-9364 (2008).

6. A. P. Rudhall et al., "Exploring the ultrashort pulse laser parameter space for membrane permeabilisation in mammalian cells," Sci. Rep. 2, 858 (2012).

7. M. Antkowiak et al., "Femtosecond optical transfection of individual mammalian cells," Nat. Protoc. 8(6), 1216-1233 (2013).

8. A. A. Davis et al., "Optoporation and genetic manipulation of cells using femtosecond laser pulses," Biophys. J. 105(4), 862-871 (2013).

9. H. G. Breunig et al., "High-throughput continuous flow femtosecond laser-assisted cell optoporation and transfection," Microsc. Res. Tech. 77(12), 974-979 (2014).

10. H. G. Breunig et al., "Software-aided automatic laser optoporation and transfection of cells," Sci. Rep. 5, 11185 (2015).

11. K. König and U. Tirlapur, "Method for transferring molecules in vital cells by means of laser beams and arrangement for carrying out said method," Patent No. US7892837 B2 (2002).

12. R. R. Alfano and C. H. Liu, "Photon-mediated introduction of biological materials into cells and/or cellular components," Patent No. US6346101 B1 (1997).

13. A. Uchugonova et al., "Optical reprogramming and optical characterization of cells using femtosecond lasers," Chapter 13, in Optically Induced Nanostructures, K. König and A. Ostendorf, Eds., de Gruyter, Berlin (2015).

14. A. Uchugonova et al., "Optical reprogramming of human cells in an ultrashort femtosecond laser microfluidic transfection platform," $J$. Biophotonics (2015). [Epub ahead of print]

15. Z. Földes-Papp et al., "Trafficking of mature miRNA-122 into the nucleus of live liver cells," $C P B$ 10(6), 569-578 (2009).

16. A. Vogel et al., "Mechanisms of femtosecond laser nanosurgery of cells and tissues," Appl. Phys. B 81(8), 1015-1047 (2005).

17. O. Nadiarnykh et al., "Carcinogenic damage to deoxyribonucleic acid is induced by near-infrared laser pulses in multiphoton microscopy via combination of two- and three-photon absorption," J. Biomed. Opt. 17(11), 116024 (2012). 